platelets from SLE patients. MS analysis revealed 32 proteins with $\geq 1.5$-fold difference and a p-value of less than 0.05 (Abundance Ratio Adjusted). STAT1, ISG15, NMI and TRIM25 were among 19 proteins expressed at higher levels in small platelets and unbiased enrichments analyses showed a significant overrepresentation of proteins related to type I interferon signaling.

Conclusions The increased mitochondrial depolarization in platelets from SLE patients is an indication but not conclusive evidence of increased platelet apoptosis. Interestingly, decreased sized platelets from SLE patients showed an up regulation of type I interferon related proteins, suggesting direct or indirect influence of IFN. This is a novel finding that may suggest that platelet size is related to IFN signaling. Further studies will be conducted to investigate the mechanistic and potential clinical role of this finding.

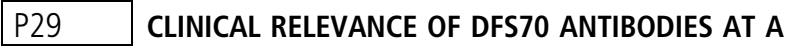 COMMUNITY HOSPITAL}

${ }^{1}$ Ana Rita Maia, ${ }^{2}$ Ana Sofia Pinto, ${ }^{2,3}$ Joana Ferreira, ${ }^{2,3}$ Cláudia Vaz. ${ }^{1}$ ULS Guarda, Guarda; ${ }^{2}$ Dept. Rheumatology, ULS Guarda, Guarda; ${ }^{3}$ Faculty of Health Sciences, University of Beira Interior, Covilhã, Portugal

10.1136/lupus-2020-eurolupus.77

Background/Purpose Antinuclear antibodies (ANA) are a serological hallmark of systemic autoimmune rheumatic diseases (SARD), such as systemic lupus erythematosus (LES), Sjogren's syndrome, systemic sclerosis and mixed connective tissue disease. Indirect immunofluorescence using human epithelial cells (HEp-2) is the gold standard for ANA screening. The nuclear pattern dense fine speckled (DFS) is one of the most common found when ANA levels are increased. DFS70 antibodies have been detected in inflammatory and neoplastic diseases; in contrast they are rare in SARD. These findings lead to research about their clinical implications. The aims of this study are: understand the meaning of anti-DFS70 antibodies; recognize the relevance of these antibodies in the diagnosis of patients with increased ANA titers in the context of SARD and non-rheumatic pathology (NRP).

Methods A retrospective observational study of consecutive patients observed during 2018 in a community hospital with anti-DFS70 antibodies positivity were performed. Sex, age, clinical and autoantibodies associations were recorded. Ethical approval was obtained and it was executed in compliance with the Helsinki Declaration.

Results Of forty-seven patients 38 (80.9\%) were females; mean of age was 51,57 years; $10(21.3 \%)$ were diagnosed with SARD (among which 4 were LES, 5 rheumatoid arthritis and 1 had autoimmune thyroiditis); 10 (21.3\%) NRP (2 with asthma, 1 allergic rhinitis, 1 sinusitis, 1 urticaria, 1 vitiligo, 2 neoplasia and 2 Gilbert's syndrome) and 27 (57.4\%) had no pathology associated - 4 patients of them presented positive antibodies without SARD.

Conclusions Although DFS70 antibodies are not specific for a particular condition, our study shows that they are a useful biomarker for differentiating between SARD and NRP when others antibodies are presented and it should be considered as a negative predictor for SARD if no other antibody is present. Whereby, DFS70 should be integrated into ANAs initial interpretation algorithm to avoid further studies.

\section{P30 A CUSTOM-MADE MICROARRAY FOR DETECTION OF AUTOANTIBODIES IN SYSTEMIC LUPUS ERYTHEMATOSUS}

${ }^{1}$ Linda Mathsson-Alm, ${ }^{1}$ Kerstin Anger, ${ }^{1}$ Jorge Dias, ${ }^{2}$ Henny Otten, ${ }^{3}$ Tammo Brunekreef, ${ }^{4}$ Sascha Swiniarski, 'Maryam Poorafshar. ${ }^{1} T$ Thermo Fisher Scientific, Uppsala, Sweden; ${ }^{2}$ Center of Translational Immunology, UMC Utrecht, Utrecht, The Netherlands; ${ }^{3}$ Dept. of Rheumatology and Clinical Immunology, UMC Utrecht, Utrecht, The Netherlands; ${ }^{4}$ Thermo Fisher Scientific, Freiburg, Germany

\subsection{6/lupus-2020-eurolupus.78}

Background Systemic Lupus Erythematosus (SLE) is a heterogeneous autoimmune disease associated with chronic inflammation with progressive organ degeneration. Early diagnosis intervention is crucial to improve overall disease course. AntidsDNA antibodies are the most sensitive serological biomarkers for SLE. The available diagnostic methods, however, identify different antibody subpopulations against dsDNA leading to varying performance. Thus, a serological biomarker profile specific to aid timely diagnosis and sensitive for disease activity of SLE remains an important clinical unmet need.

In this study a microarray was developed that enabled a simple and rapid characterization of the serological profile of SLE patients. In this microarray it was possible to analyze up to 90 autoantibodies in $<10 \mu \mathrm{l}$ serum, making it a useful tool for characterization of SLE patient cohorts.

Methods A microarray was developed by spotting autoantigens in an arrayed fashion onto the surface of maxisorp plastic slides. Binding of each antigen was optimized regarding concentration and spotting buffer. The assay was performed by incubating diluted serum on the array followed by incubation with a fluorescent-labeled anti-human IgG antibody. Fluorescence intensities were measured with a laser scanner and the resulting images analyzed with an image analysis software. The microarray performance was compared to single analytes on EliA $^{\mathrm{TM}}$ (Phadia AB, Uppsala, Sweden). For technical evaluation serum from 160 SLE patients and 313 healthy blood donors were measured.

Results Overall, there was strong correlations between microarray and EliA results for relevant biomarkers. The mean CV\% between productions, lots and assays were 11,8 and $7 \%$, respectively.

Conclusions The developed microarray showed good technical performance and is a promising tool for the characterization of the serological profile of SLE patients. Furthermore, it has been applied in a clinical study as a discovery tool for new autoantibodies and patterns of autoantibodies with promising results.

\section{P31 ANTI-DOMAIN I POSITIVITY IN SLE AT DIAGNOSIS IS PREDICTIVE OF ATHEROSCLEROTIC PLAQUE DEVELOPMENT}

${ }^{1}$ Thomas McDonnell, ${ }^{1}$ Filipa Farinha, ${ }^{2}$ Charis Pericleous, ${ }^{3}$ Maura Griffin, ${ }^{3}$ Andrew Nicholaides, 'Sara Croca, 'Ian Giles, 'Anisur Rahman. ${ }^{1}$ Rheumatology, Division of Medicine, University College London, London; ${ }^{2}$ Vascular Dept., Imperial College London, London; ${ }^{3}$ Vascular Non-invasive Diagnostic Centre, London, UK

\subsection{6/lupus-2020-eurolupus.79}

Background Cardiovascular disease is a significant burden on SLE patients and no satisfactory markers exist to predict atherosclerotic development in patients. This study utilised an orthogonal approach to develop a marker of cardiovascular progression with predictive value in SLE patients. 